Pacific Journal of Mathematic 


\section{A HOMOLOGY SPECTRAL SEQUENCE FOR SUBMERSIONS}

\section{Patrick C. Endicott and J. WolfGang Smith}

By a submersion we shall understand a $C^{\infty}$ surjection $f$ : $X \rightarrow Y$ between paracompact $C^{\infty}$ manifolds with $\operatorname{dim} X \geq \operatorname{dim} Y$, subject to the condition that the differential of $f$ have maximal rank at all points. This implies that the fiber $f_{y}$ over any point $y \in Y$ will be a smooth regularly imbedded submanifold of $X$. Differentiable fiber bundles constitute a special class of submersions, characterized by the existence of local product structures, and in this particular case all fibers $f_{y}$ are homeomorphic to a standerd fiber $F$. The central result in the homology theory of fiber bundles asserts the existence of a convergent spectral sequence whose $E^{\infty}$ term is the bigraded group associated to some filtration of $H_{*}(X ; G)^{1}$, and for which

$$
E_{s, t}^{2} \approx H_{s}\left(Y ; H_{t}(F ; G)\right)
$$

in case the bundle is orientable over $G$. In the present paper this result is generalized to arbitrary submersions. The $E^{2}$ terms now come to be identified with certain groups $H_{s, t}(f ; G)$ representing a homology functor from the category of submersions to the category of bigraded groups, which reduce of course to $H_{s}\left(Y ; H_{t}(F ; G)\right)$ in the classical case.

The functor in question has been previously studied by Sekino [4], who has shown that in its relativised form it satisfies axioms of the Eilenberg-Steenrod type, for which a categoricity theorem can be established. In $\S 1$ we will give a direct constructive definition of the homology groups $H_{s, t}(f ; G)$. The construction of the spectral sequence and identification of its $E^{2}$ terms take place in $\S \S 2$ and 3 , followed by a final section setting forth a few applications of the theory. We also note that $\S 4$ should be entirely comprehensible following $\S 1$.

A few remarks on the subject of applications may be in order. The usefulness of a spectral sequence clearly depends on the ease with which the homology (or cohomology) groups representing its $E^{2}$ terms can be evaluated or estimated in various geometric situations. For the spectral sequence under consideration we have found that these calculations can generally be effected with particular ease and directness, and so far as the study of submersions is concerned, this approach may well offer advantages over the sheaf-theoretic cohomology approach represented by the spectral sequence of Leray. Our

1 Throughout this paper $G$ will denote an arbitrary coefficient group and $H_{*}$ the singular homology functor. 
construction capitalizes on two fundamental facts: firstly, that every compact subset of a fiber can be enclosed in tubular a neighborhood, a property which generalizes the local product structures characteristic of fiber bundles, and which itself may be regarded as characterizing the class of submersions. ${ }^{2}$ Secondly, we make stong use of the fact that homology commutes with direct limits. These premises, together with the circumstance that our base spaces are triangulable, enable one to construct a homology theory and corresponding spectral sequence constituting a particulary direct geometrical approach to the problem at hand, which is to study the relations between the topological structures of the total space, base space and fibers. To give at least one example of such a relation at this point, we will cite the following result established in $\S 4$.

THEOREM. Let $f: X \rightarrow Y$ be a submersion between orientable manifolds of dimensions $n$ and $m$, respectively, having connected fibers; and let $U$ denote the subspace of $Y$ corresponding to compact fibers. The Betti number of $X$ and $U$ are then subject to the relation $R_{n-1}(X ; G) \geqq R_{m-1}(U ; G)$.

In particular, when $Y$ is the real line this theorem asserts that $R_{n-1}(X ; G)$ is bounded below by the number of components in $U$, which might be a hitherto unnoticed result concerning real-valued functions with nowhere vanishing gradient. It should also be pointed out that our theorem pertains strictly to the theory of submersions, inasmuch as it obviously fails for more general maps (the map $f: R^{2} \rightarrow R$ defined by the formula $f(x, y)=x^{2}+y^{2}$ would provide an easy counter-example).

1. Homology of submersions. Our construction hinges on the concept of a simplicial bundle over a simplicial complex $K$, which we define to be a function that assigns to each simplex $\sigma \in K$ a commutative triangle

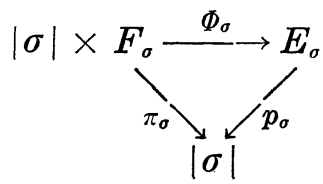

where $E_{\sigma}, F_{\sigma}$ are topological spaces, $|\sigma|$ is the closed space of $\sigma, \Phi_{\sigma}$ a homeomorphism and $\pi_{\sigma}$ the natural projection, this data being subject to the following descending face condition: for every face $\tau<\sigma$ and $x \in|\tau|$,

${ }^{2}$ From this point of view one could dispense with the assumpation of differentiability. 


$$
p_{\sigma}^{-1}(x) \subset p_{\tau}^{-1}(x)
$$

This insures that the projections $p_{\sigma}$ induce a projection $p: E \rightarrow|K|$, where $E$ denotes the topological sum of the spaces $E_{\sigma}$. A simplicial bundle is said to approximate a submersion $f: X \rightarrow Y$ with respect to a compact subspace $C \subset X$ provided $C \subset E \subset X, f(C) \subset|K| \subset Y$ and $p=f \mid E$ (the restriction of $f$ to $E$ ). The essential connection between simplicial bundles and submersions may now be expressed in terms of the following basic approximation theorem.

THEOREM 1.1. Let $f: X \rightarrow Y$ be a submersion and $C$ a compact subspace of $X$. Let $M$ be a simplicial complex such that ${ }^{3} Y=|M|$. Then there exists a simplicial bundle over some barycentric subdivision $K$ of a subcomplex of $M$ which approximates $f$ with respect to $C$, and whose total space $E$ is compact.

The proof is substantially identical ${ }^{4}$ with the proof of Lemma 2 in Smith [6] and proceeds by an elementary inductive argument involving tubular neighborhood constructions. Essentially the approximation theorem permits us to view a submersion as the direct limit of an approximating system of simplicial bundles, an idea that has been formulated in category-theoretic terms by Sekino [4] and constitues the basis of his theory. For our present purpose it will suffice to associate with any given submersion a sequence of simplicial bundles satisfying the conditions of the following theorem, conditions which insure that the given sequence is cofinal in the full approximating system and consequently adequate for the computation of direct limits.

THEOREM 1.2. Given a submersion $f: X \rightarrow Y$, there exists a sequence $\left\{{ }_{\alpha} p:{ }_{\alpha} E \rightarrow\left|{ }_{\alpha} K\right|\right\}$ of simplicial bundles such that

(1) for every compact subspace $C \subset X$ there exists a positive integer $\alpha$ such that ${ }_{\alpha} p:\left.{ }_{\alpha} E \rightarrow\right|_{\alpha} K \mid$ approximates $f$ with respect to $C$;

(2) for $\alpha<\beta,{ }_{\alpha} E \subset{ }_{\beta} E$ and there exists a nonnegative integer $n$ such that ${ }_{a}^{n} K \subset{ }_{\beta} K$ (where ${ }^{n} K$ denotes the $n$th barycentric subdivision of $K$ ).

${ }^{3}$ Our differentiable manifolds are triangulable, and we will suppress the triangulating homeomorphism by assuming the corresponding identifications.

${ }_{4}$ The only difference being that in [6] we were concerned with simplicial bundles "dual" to the bundled here envisaged, i.e., bundles for which our descending face condition was replaced by a corresponding ascending face condition. While simplicial bundles of the descending type are adapted to homology theory, bundles of the ascending types are adapted to cohomology and obstruction theories. The only change required to adapt the proof in [6] to the present case is to reverse the direction of the induction procedure so as to go from high to low dimensions. See also Endicott [2], pp. 39-43. 
To prove this on the basis of our first approximation theorem, one need only choose a covering of $X$ in the form of a nested sequence $\left\{C_{\alpha}\right\}$ of a compact subsets and proceed by induction. Thus we suppose that for all $\alpha \leqq \gamma$ we have simplicial bundles $p_{\alpha}:{ }_{\alpha} E \rightarrow\left|{ }_{\alpha} K\right|$ satisfying condition (1) and (2), with the additional stipulation that each ${ }_{\alpha} K$ is a barycentric subdivion of a subcomplex belonging to a given triangulation $L$ of $Y$. Let $m$ be a nonnegative integer such that ${ }^{m} K_{\gamma} \subset L$. Applying 1.1 with $C=C_{\gamma+1} \cup_{\gamma} E$ and $M={ }^{m} L$ yields a simplicial bundle on the $(\gamma+1)$-level which preserves our inductive hypothesis, and this completes the proof.

At this point the fundamental idea underlying our construction of the homology groups $H_{s, t}(f ; G)$ can be explained. Given a simplicial bundle $p: E \rightarrow|K|$, coefficient group $G$ and integer $t$, we will define a corresponding chain complex $C_{*}\left(K ; H_{t}(F ; G)\right)$ in which the homology groups $\left\{H_{t}\left(F_{\sigma} ; G\right), \sigma \in K\right\}$ function as a local coefficient system. Given a submersion $f: X \rightarrow Y$, we will choose an approximating sequence of simplicial bundles in accordance with 1.2, and this gives rise to a corresponding sequence of chain complexes $C_{*}\left({ }_{\alpha} K ; H_{t}\left({ }_{\alpha} F ; G\right)\right)$, together with chain projections $\phi_{\alpha}^{\beta}$ defined for $\alpha<\beta$, constituting a direct system of chain complexes. The homology groups in question may now be defined by setting

$$
\left(H_{s, t}(f ; G)\right)=H_{s}\left(\lim _{\rightarrow} C_{*}\left({ }_{\alpha} K ; H_{t}\left({ }_{\alpha} F ; G\right)\right)\right) ;
$$

or equivalently, as the direct limit of the direct system

$$
\left\{H_{s}\left(C_{*}\left({ }_{\alpha} K ; H_{t}\left({ }_{\alpha} F ; G\right)\right)\right), \phi_{\alpha^{*}}^{\beta}\right\} \text {. }
$$

It follows from the previously noted cofinality of our approximating sequence $\left\{{ }_{\alpha} p:{ }_{\alpha} E \rightarrow{ }_{\alpha} K \mid\right\}$ that the groups $H_{s, t}(f ; G)$ can be identified with the functorial homology groups investigated by Sekino, a fact which we need not presuppose, however, and which will play no role in the applications presented in $\S 4$.

We proceed now to define the chain complex associated with a simplicial bundle $p: E \rightarrow|K|$. For $s \geqq 0$ we let

$$
C_{s}\left(K ; H_{t}(F ; G)\right)=\bigoplus_{\sigma \in K^{(s)}} H_{s}(|\sigma|,|\dot{\sigma}|) \otimes H_{t}\left(F_{\sigma} ; G\right)
$$

where $K^{(s)}$ denotes the set of $s$-simplexes in $K$ and $|\dot{\sigma}|$ the boundary of $|\sigma|$. To obtain boundary operators, we note that the inclusion $\operatorname{maps}^{5}$

$$
j_{o}:(|\sigma|,|\dot{\sigma}|) \subset\left(\left|K^{s}\right|,\left|K^{s-1}\right|\right)
$$

induce a direct sum representation

${ }^{5} K^{8}$ denotes the $s$-skeleton of $K$. 


$$
\left\{j_{\sigma^{*}}\right\}: \bigoplus_{\sigma \in K^{(s)}} H_{s}(|\sigma|,|\dot{\sigma}|) \approx H_{s}\left(K^{s}, K^{s-1}\right),
$$

and we let

$$
q_{\sigma}: H_{s}\left(K^{s}, K^{s-1}\right) \longrightarrow H_{s}(|\sigma|,|\dot{\sigma}|)
$$

denote the corresponding projections. For every $\tau \in K^{(s-1)}$ we can define a homomorphism

$$
\varepsilon_{\sigma}^{\tau}: H_{s}(|\sigma|,|\dot{\sigma}|) \longrightarrow H_{s-1}(|\tau|,|\dot{\tau}|)
$$

by setting $\varepsilon_{\sigma}^{\tau}=q_{\tau} \circ \partial_{*} \circ j_{\sigma^{*}}$, where

$$
\partial_{*}: H_{s}\left(K^{s}, K^{s-1}\right) \longrightarrow H_{s-1}\left(K^{s-1}, K^{s-2}\right)
$$

denotes the connecting homomorphism for the triple $\left(K^{s}, K^{s-1}, K^{s-2}\right)$. A simple direct calculation gives

Lemma 1.4. Let $\tau$ and $\rho$ denote simplexes in $K$ of dimensions $(s-1)$ and $(s+1)$, repectively. Then

$$
\sum_{\sigma \in K^{(s)}} \varepsilon_{\sigma}^{\tau} \circ \varepsilon_{\rho}^{\sigma}=0
$$

For $\tau<\sigma$ and $x \in|\tau|$ one can define (by virtue of the descending face condition) an injection $i_{\sigma, x}^{\tau}: F_{\sigma} \rightarrow F_{\tau}$ through the formula

$$
i_{\sigma, x}^{\tau}(y)=\pi_{\tau}^{\prime} \circ \Phi_{\tau}^{-1} \circ \Phi_{\sigma}(x, y)
$$

where $\pi_{\tau}^{\prime}:|\tau| \times F_{\tau} \rightarrow F_{\tau}$ denotes the natural projection. Since the homotopy class of $i_{\sigma, x}^{\tau}$ is clearly independent of $x$, one obtains canonical homomorphisms

$$
i_{\sigma}^{\tau}: H_{*}\left(F_{\sigma} ; G\right) \longrightarrow H_{*}\left(F_{\tau} ; G\right)
$$

henceforth referred to as fiber projections, for which the following transitivity condition is easily verified [4, p. 39]:

$$
i_{\sigma}^{\tau} \circ i_{\rho}^{\sigma}=i_{\rho}^{\tau} \text { for } \tau<\sigma<\rho .
$$

The desired boundary operators

$$
\text { o: } C_{s}\left(K ; H_{t}(F ; G)\right) \longrightarrow C_{s-1}\left(K ; H_{t}(F ; G)\right)
$$

may now be defined for $s \geqq 0$ through the formula ${ }^{6}$

$$
\partial(a \otimes c)=\sum_{\tau \in K^{(s-1)}} \varepsilon_{o}^{\tau}(a) \otimes i_{o}^{\tau}(c)
$$

where

\footnotetext{
${ }^{6}$ Since $\varepsilon_{\sigma}^{\tau}=0$ unless $\tau<\sigma$, the formula makes sense.
} 


$$
a \otimes c \in H_{s}(|\sigma|,|\dot{\sigma}|) \otimes H_{t}\left(F_{\sigma} ; G\right),
$$

and it follows by (1.4) and (1.6) that $\partial \circ \partial=0$.

This completes our construction of the homology groups $H_{s}\left(K ; H_{t}(F ; G)\right)$ associated with a simplicial bundle. It should be pointed out, however, that for a simplicial bundle approximating a submersion $f: X \rightarrow Y$, the fiber projections $i_{\sigma}^{\tau}$ admit a simple geometric interpretation. The homeomorphisms $\Phi_{\sigma}:|\sigma| \times F_{\sigma} \rightarrow E_{\sigma}$ now constitute tubular neighborhoods in $X$, and without loss of generality one may assume that these tubular neighborhoods are associated with a given "horizontal distribution" on $X$, i.e., a distribution of $m$-planes which is transverse to the fibers. If for each $\sigma \in K$ we identify $F_{\sigma}$ with $p_{\sigma}^{-1}\left(b_{\sigma}\right)$, where $b_{\sigma}$ is (say) the barycenter of $\sigma$, then it turns out that the fiber projection $i_{\sigma}^{\tau}$ is induced by an injection $i: F_{\sigma} \rightarrow F_{\tau}$ induced by the horizontal liftings of some path in $|\sigma|$ from $b_{\sigma}$ to $b_{\tau}$.

It remains now to construct the chain projections $\phi_{\alpha}^{\beta}$ associated with an approximating sequence (1.2), for which purpose we need to consider canonical (or "barycentric") subdivisions of simplicial bundles [4, p. 42]. Let $p: E \rightarrow|K|$ be a simplicial bundle. For each $s$-simplex $\omega$ belonging to the $n$th barycentric subdivision ${ }^{n} K$ of $K$ there exists a unique $\sigma \in K^{(s)}$ such that $|\omega| \subset|\sigma|$, and setting ${ }^{n} F_{\omega}=F_{\sigma},{ }^{n} E_{\omega}=$ $p_{\sigma}^{-1}(|\omega|),{ }^{n} \Phi_{\omega}=\Phi_{\sigma}|| \omega \mid \times F_{\sigma}$ and ${ }^{n} p_{\omega}=p_{\sigma} \mid{ }^{n} E_{\omega}$ one obtains a new simplicial bundle ${ }^{n} p: E \rightarrow\left|{ }^{n} K\right|$, which is the $n$th canonical subdivision. On the chain level one obtains a corresponding chain homomorphism

$$
S d^{n}: C_{s}\left(K ; H_{t}(F ; G)\right) \longrightarrow C_{s}\left({ }^{n} K ; H_{t}\left({ }^{n} F ; G\right)\right)
$$

through the formula

$$
S d^{n}(a \otimes c)=S d_{\sigma}^{n}(a) \otimes c
$$

where again $a \otimes c$ is given as in (1.8), and

$$
S d_{\sigma}^{n}: H_{s}(|\sigma|,|\dot{\sigma}|) \longrightarrow \bigoplus H_{s}(|\omega|,|\dot{\omega}|)
$$

is essentially the classical subdivision operator, which may be defined as follows. Let $A=\left|{ }^{n} K^{s-1}\right| \cap|\sigma|$, and let $i:(|\sigma|,|\dot{\sigma}|) \subset(|\sigma|, A)$ and $i_{\sigma}^{\omega}:(|\omega|,|\dot{\omega}|) \subset(|\sigma|, A)$ denote inclusions. Then

$$
\left\{i_{o^{*}}^{\omega}\right\}: \bigoplus H_{s}(|\omega|,|\dot{\omega}|) \approx H_{s}(|\sigma|, A)
$$

constitutes a direct-sum representation [8, p. 474], and one has

$$
S d_{o}^{n}=\left\{i_{\sigma^{*}}^{\omega}\right\}^{-1} \circ i_{*} \equiv \sum_{\omega} B_{o, \omega}^{n} .
$$

Now let $\left\{{ }_{\alpha} p:{ }_{\alpha} E \rightarrow\left|{ }_{\alpha} K\right|\right\}$ denote an approximating sequence as given by 1.2 , and let $\alpha<\beta$. This determines an integer $n$ such that ${ }_{\alpha}^{n} K \subset{ }_{\beta} K$. Moreover, for every $\sigma \epsilon_{\alpha} K^{(s)}$ and $\omega \in_{\beta} K^{(s)}$ with $|\omega| \subset|\sigma|$, 
we may identify ${ }_{\alpha} F_{\sigma}$ and ${ }_{\beta} F_{\omega}$ with ${ }_{\alpha} p_{\sigma}^{-1}(x)$ and ${ }_{\beta} p_{\omega}^{-1}(x)$, respectively, for some $x \in|\omega|$. Since ${ }_{\alpha} E \subset E_{\beta}$, one thus obtains an inclusion induced injection $j_{\sigma}^{\omega}:{ }_{\alpha} F_{\sigma} \rightarrow{ }_{\beta} F_{\omega}$, whose homotopy class is independent of $x$. The desired chain projections

$$
\phi_{\alpha}^{\beta}: C_{s}\left({ }_{\alpha} K ; H_{t}\left({ }_{\alpha} F ; G\right)\right) \longrightarrow C_{s}\left({ }_{\beta} K ; H_{t}\left({ }_{\beta} F ; G\right)\right)
$$

are defined on the generators $a \otimes c$ by setting

$$
\phi_{\alpha}^{\beta}(a \otimes c)=\sum_{\omega} B_{o, \omega}^{n}(a) \otimes j_{\sigma^{*}}^{\omega}(c),
$$

and it is obvious that the transitivity conditions for a direct system are satisfied.

2. The spectral sequence of a simplicial bundle. As previously affirmed, we shall define a spectral sequence corresponding to a given submersion $f: X \rightarrow Y$ whose $E_{s, t}^{2}$ term may be identified with the homology group $H_{s, t}(f ; G)$ constructed in the course of $\S 1$. Again our procedure will involve two basic steps: firstly, we will define a spectral sequence associated with a simplicial bundle $p: E \rightarrow|K|$, whose $E_{s, t}^{2}$ term may be identified with the corresponding homology group $H_{s}\left(K ; H_{t}(F ; G)\right)$. In $\S 3$ we shall once more consider an approximating sequence of simplicial bundles, which gives rise to a direct system of spectral sequences, from which we obtain the desired spectral sequence in the direct limit.

Our first step follows an approach introduced by Chern and Spanier [1], and subsequently extended by Spanier [8], which considers the filtration of the total space $X$ induced from the skeletal filtration resulting from a triangulation of the base space. This filtration of $X$ induces a filtration ${ }^{7}$ of the singular chain complex $S(X ; G)$, which in turn gives rise to a spectral sequence [8; §§9.1 \& 9.2]. Applying this idea to the case of a simplicial bundle $p: E \rightarrow|K|$, we let $E_{s}$ denote the empty set for $s<0$ and set

$$
E_{s}=\bigcup_{\sigma \in K^{s}} E_{\sigma} \text { for } s \geqq 0 ;
$$

to obtain the following result, which is a special case of Theorem 2 [8; p. 469].

THEOREM 2.1. There exists a convergent $E^{1}$ spectral sequence with

$$
E_{s t}^{1} \approx H_{s+t}\left(E_{s}, E_{s-1} ; G\right),
$$

$d^{1}$ corresponding to the connecting homomorphism $\partial_{*}$ of the triple

7 This filtration is convergent and bounded below. 
$\left(E_{s}, E_{s-1}, E_{s-2}\right)$, and $E^{\infty}$ isomorphic to the bigraded group associated to the filtration of $H_{*}(E ; G)$ defined by

$$
F_{s} H_{*}(E ; G)=\operatorname{Im}\left[H_{*}\left(E_{s} ; G\right) \longrightarrow H_{*}(E ; G)\right] \text {. }
$$

It will be shown that there exist isomorphisms

$$
\psi_{s}: C_{s}\left(K ; H_{t}(F ; G)\right) \approx H_{s+t}\left(E_{s}, E_{s-1} ; G\right)
$$

under which the boundary operators (1.7) correspond precisely to $\partial_{*}$. This fact, together with 2.1, will give our first main result,

THEOREM 2.2. Given a simplicial bundle $p: E \rightarrow|K|$ and coefficient group $G$, there exists a convergent $E^{2}$ spectral sequence with

$$
E_{s, t}^{2} \approx H_{s}\left(K ; H_{t}(F ; G)\right),
$$

and $E^{\infty}$ isomorphic to the bigraded group associated with the filtration of $H_{*}(E ; G)$ described in 2.1 .

We begin our construction ${ }^{8}$ of $\psi_{s}$ with

Lemma 2.3. For every $\sigma \in K^{(s)}$ the homology cross product defines an isomorphism

$$
\mu_{\sigma}: H_{s}(|\sigma|,|\dot{\sigma}|) \otimes H_{t}\left(F_{\sigma} ; G\right) \approx H_{s+t}\left((|\sigma|,|\dot{\sigma}|) \times F_{\sigma} ; G\right) .
$$

This follows directly from the Künneth formula [8;10, p. 235]. Next, the homeomorphism $\Phi_{\sigma}$ of the simplicial bundle furnishes as isomorphism

$$
\Phi_{\sigma^{*}}: H_{*}\left((|\sigma|,|\dot{\sigma}|) \times F_{\sigma} ; G\right) \approx H_{*}\left(E_{\sigma}, E_{\dot{\sigma}} ; G\right)
$$

where $E_{\dot{\sigma}}=p_{\sigma}^{-1}(|\dot{\sigma}|)$. The third step is given by

LEMma 2.4. The inclusion

$$
i_{\sigma}^{\prime}:\left(E_{o}, E_{\dot{\sigma}}\right) \subset p^{-1}(|\sigma|,|\dot{\sigma}|)
$$

induces an isomorphism between corresponding homolgy groups.

To prove this, let $e$ denote a closed $s$-cell in the interior of $|\sigma|$, let $\stackrel{e}{e}$ denote the interior of $e$, and consider the commutative diagram ${ }^{9}$

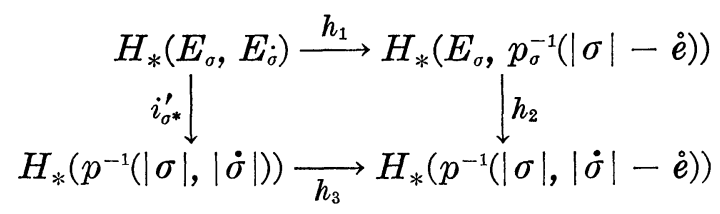

${ }^{8}$ Here one should recall (1.3).

${ }^{9}$ For brevity we will suppress $G$ in some diagrams. 
induced by inclusion maps. Since $E_{\sigma}$ and $p^{-1}(|\sigma|)$ can differ only over $|\dot{\sigma}|$, and $|\dot{\sigma}|$ lies in the interior of $(|\sigma|-\grave{e})$, one sees that $h_{2}$ is an excision induced isomorphism. Moreover, since $|\dot{\sigma}|$ is obviously a deformation retract of $(|\sigma|-\ddot{e})$, the isomorphism $\Phi_{\sigma}$ guarantees that $E_{\dot{\sigma}}$ is likewise a deformation retract of $p_{\sigma}^{-1}(|\sigma|-\varrho)$, which implies that $h_{1}$ is an isomorphism. But since any deformation retraction

$$
r_{\sigma}: p_{\sigma}^{-1}\left(|\sigma|-\stackrel{e}{)} \longrightarrow E_{\sigma}\right.
$$

extends trivially to a deformation retraction

$$
r_{\sigma}^{\prime}: p^{-1}(|\sigma|-e) \longrightarrow p^{-1}(|\dot{\sigma}|),
$$

it also follows that $h_{3}$ is an isomophism, and this proves our lemma.

The final step in our construction of $\psi_{s}$ is provided by the following result, which is an exact analogue of Lemma 2 [8; p. 474].

Lemma 2.6. The inclusions

$$
i_{\sigma}^{\prime \prime}: p^{-1}(|\sigma|,|\dot{\sigma}|) \subset\left(E_{s}, E_{s-1}\right)
$$

induce a direct-sum representation

$$
\left\{i_{\sigma^{*}}^{\prime \prime}\right\}: \underset{\sigma \in K^{(s)}}{\bigoplus_{*}} H_{*}\left(p^{-1}(|\sigma|,|\dot{\sigma}|) ; G\right) \approx H_{*}\left(E_{s}, E_{s-1} ; G\right) .
$$

Moreover, in view of the deformation retractions (2.5), Spanier's proof carries over verbatim to the present case.

Combining the isomorphisms established in the four preceding steps, we may now define $\psi_{s}$ by the formula

$$
\dot{\psi}_{s}=\left\{i_{\sigma^{*}}\right\} \circ\left[\bigoplus \Phi_{\sigma^{*}}\right] \circ\left[\bigoplus \mu_{\sigma}\right]
$$

where the direct sums extend over all $\sigma \in K^{(s)}$ and $i_{\sigma}=i_{\sigma}^{\prime \prime} \circ i_{\sigma}^{\prime}$ denotes the inclusion $\left(E_{\sigma}, E_{\dot{\sigma}}\right) \subset\left(E_{s}, E_{s-1}\right)$.

It remains to be shown that the isomorphisms $\psi_{s}$ commute with the respective boundary operators. Before embarking upon this somewhat delicate calculation, we shall establish the following lemma, which turns out to be crucial.

Lemma 2.8. Let $\sigma \in K^{(s)}$ and $\tau$ an $(s-1)$-face of $\sigma$. Let $x_{0} \in|\tau|$, and let

$$
\Phi_{\sigma}^{:}:(|\tau|,|\dot{\tau}|) \times F_{\sigma} \longrightarrow\left(E_{\sigma}, E_{\dot{\sigma}}\right)
$$

denote the map induced by $\Phi_{\sigma}$. There exists a homotopy

$$
H:(|\tau|,|\dot{\tau}|) \times F_{\sigma} \times I \longrightarrow\left(E_{s}, E_{s-1}\right)
$$


such that

$$
H: i_{=} \circ \Phi_{\tau} \circ\left(1_{\tau} \times i_{\sigma, x_{0}}^{\tau}\right) \cong i_{\sigma} \circ \Phi_{\sigma}^{\tau}
$$

where $1_{-}:(|\tau|,|\dot{\tau}|) \rightarrow(|\tau|,|\dot{\tau}|)$ is the identity and $i_{\sigma}^{\tau} x_{0}: F_{o} \rightarrow F_{\tau}$ the injection given by (1.5).

To construct $H$, we first define a map

$$
\rho:(|\tau|,|\dot{\tau}|) \times F_{\sigma} \longrightarrow(|\tau|,|\dot{\tau}|) \times F_{s}
$$

by setting

$$
\rho(x, y)=\left(x, i_{\sigma, x}^{\tau}(y)\right) .
$$

One verifies by a simple direct calculation that

$$
\Phi_{\circ} \circ \rho=j \circ \Phi_{\sigma}^{-}
$$

where

$$
j: p_{\sigma}^{-1}(|\tau|,|\dot{\tau}|) \subset\left(E_{\tau}, E_{:}\right)
$$

denotes the inclusion. Composing both sides of (2.9) with $i_{\text {, }}$, and observing that $i_{=} \circ j$ is a restriction of $i_{\sigma}$, one obtains

$$
i_{\tau} \circ \Phi_{\tau} \circ \rho=i_{\sigma} \circ \Phi_{\sigma}^{-} .
$$

It will therefore suffice to construct a homotopy

$$
\bar{H}:(|\tau|,|\dot{\tau}|) \times F_{\sigma} \times I \longrightarrow(|\tau|,|\dot{\tau}|) \times F_{\tau}
$$

such that

$$
\bar{H}: \rho \cong 1_{\tau} \times i_{\bar{\sigma}, x_{0}}^{\bar{c}}
$$

from which the desired homotopy $H$ can be obtained by setting $H=i_{\tau} \circ \Phi_{\tau} \circ \bar{H}$. To construct $\bar{H}$, we observe that there exists for each $x \in|\tau|$ an obvious homotopy $H_{x}: F_{\sigma} \times I \rightarrow F_{\tau}$ such that

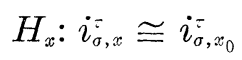

which results from (1.5) by identifying $I$ with the line segment in $|\tau|$ from $x$ to $x_{0}$ under the canonical affine map. One can then define $\bar{H}$ through the formula

$$
\bar{H}(x, y, t)=\left(x, H_{x}(y, t)\right)
$$

which gives (2.10), and establishes our lemma.

The desired commutativity reduces to the formula

$$
\psi_{s-1} \circ \partial(a \otimes c)=\partial_{*} \cdot \psi_{s}(a \otimes c)
$$

which we will now establish by direct calculation. Since $\partial$ is given by (1.7), one obtains 


$$
\psi_{s-1} \cdot \partial(a \otimes c)=\psi_{s-1} \sum_{\tau} \varepsilon_{\sigma}^{\tau}(a) \otimes i_{\sigma}^{\tau}(c)
$$

where the sum can be thought of as extending over all $\tau \in K^{(s-1)}$. Applying (2.7), and bearing in mind that $\mu_{\tau}$ represents the homology cross product, one may rewrite (2.12) in the form

$$
\psi_{s-1} \circ \partial(a \otimes c)=\sum_{\tau} i_{\tau^{*}} \cdot \Phi_{\tau^{*}}\left(\varepsilon_{\sigma}^{\tau}(a) \times i_{\sigma}^{\tau}(c)\right) .
$$

It follows from our definition of the fiber projections (given in $\S 1$ ) that

$$
i_{\sigma}^{\tau}=\left(i_{\sigma, x_{0}}^{\tau}\right)_{*}
$$

for any $x_{0} \in|\tau|$, which together with a well-known property of the cross product $[8 ; 11$, p. 235$]$ gives

$$
\varepsilon_{\sigma}^{\tau}(a) \times i_{\sigma}^{\tau}(c)=\left(1_{\tau} \times i_{\sigma, x_{0}}^{\tau}\right)_{*}\left(\varepsilon_{\sigma}^{\tau}(a) \times c\right) .
$$

Substituting this into (2.13) and applying (2.8) gives

$$
\psi_{s-1} \circ \partial(a \otimes c)=\sum_{\tau} i_{\sigma^{*}} \circ \Phi_{\sigma^{*}}^{\tau}\left(\varepsilon_{\sigma}^{\tau}(a) \times c\right) .
$$

Before going further, we must introduce some additional spaces and maps. We will let $|\ddot{\sigma}|$ denote the union of all closed $(s-2)$-faces in $\sigma$, and set $E_{\sigma}=p_{\sigma}^{-1}(|\ddot{\sigma}|)$. This brings into play the new inclusions

$$
\begin{gathered}
i_{\dot{\sigma}}:\left(E_{\dot{\sigma}}, E_{\ddot{\sigma}}\right) \subset\left(E_{s-1}, E_{s-2}\right), \\
i^{\tau}:(|\tau|,|\dot{\tau}|) \subset(|\dot{\sigma}|,|\ddot{\sigma}|),
\end{gathered}
$$

together with the homeomorphism

$$
\Phi_{\dot{\sigma}}:(|\dot{\sigma}|,|\ddot{\sigma}|) \times F_{\sigma} \approx\left(E_{\dot{\sigma}}, E_{\ddot{\sigma}}\right)
$$

constituting a restriction of $\Phi_{\sigma}$. Since

$$
i_{\sigma} \circ \Phi_{\sigma}^{\tau}=i_{\dot{\sigma}} \circ \Phi_{\dot{\sigma}} \circ\left(i^{\tau} \times 1_{F_{\sigma}}\right)
$$

one may rewrite (2.14) in the form

$$
\psi_{s-1} \circ \partial(a \otimes c)=\sum_{\tau} i_{\dot{\sigma}^{*}} \circ \Phi_{\dot{\sigma}^{*}} \circ\left(i^{\tau} \times 1_{F_{\sigma}}\right)_{*}\left(\varepsilon_{\sigma}^{\tau}(a) \times c\right) .
$$

Using $[8 ; 11$, p. 235] once more, one has

$$
\left(i^{\tau} \times 1_{F_{\sigma}}\right)_{*}\left(\varepsilon_{\sigma}^{\tau}(a) \times c\right)=i_{*}^{\tau} \circ \varepsilon_{\sigma}^{\tau}(a) \times c .
$$

By a tedious but elementary calculation, for which we refer to [2; pp. 36-37], one verifies that

$$
\sum_{\tau} i_{*}^{\tau} \circ \varepsilon_{\sigma}^{\tau}(a)=\partial_{*}^{\prime}(a)
$$


where

$$
\partial_{*}^{\prime}: H_{s}(|\sigma|,|\dot{\sigma}|) \longrightarrow H_{s-1}(|\dot{\sigma}|,|\ddot{\sigma}|)
$$

denotes the connecting homomorphism of the triple $(|\sigma|,|\dot{\sigma}|,|\ddot{\sigma}|)$. Letting

$$
\partial_{*}^{\prime \prime}: H_{s+t}\left(E_{\sigma}, E_{\tilde{\sigma}} ; G\right) \longrightarrow H_{s+t-1}\left(E_{\dot{\sigma}}, E_{\tilde{\sigma}} ; G\right)
$$

denote the connecting homomorphism of the triple $\left(E_{\sigma}, E_{\dot{\sigma}}, E_{\sigma}\right)$, one obtains

$$
\partial_{*}^{\prime}(a) \times c=\partial_{*}^{\prime \prime}(a \times c)
$$

which follows as a special case of [8; 15, p. 235], bearing in mind that the exact sequence of a triple constitutes a special case of the relative Mayer-Vietoris sequence [8; pp. 180-190]. Combining (2.16), (2.17), and (2.17), and (2.18) with (2.15) gives

$$
\psi_{s-1} \circ \partial(a \otimes c)=i_{\dot{\sigma}^{*}} \circ \Phi_{\dot{\sigma}^{*}} \circ \partial_{*}^{\prime \prime}(a \times c) .
$$

Now let $\partial_{*}^{\prime \prime \prime}$ denote the connecting homomorphism of the triple $(|\sigma|,|\dot{\sigma}|,|\ddot{\sigma}|) \times F_{\sigma}$. By naturality of the connecting homomorphism one obtains

$$
\Phi_{\sigma^{*}} \circ \partial_{*}^{\prime \prime}=\partial_{*}^{\prime \prime \prime} \circ \Phi_{\sigma^{*}}
$$

and for the same reason

$$
i_{\dot{\sigma}^{*}} \circ \partial_{*}^{\prime \prime \prime}=\partial_{*} \circ i_{\sigma_{*}} .
$$

Substituting (2.20) and (2.21) into (2.19), one obtains

$$
\psi_{s-1} \circ \partial(a \otimes c)=\partial_{*} \circ i_{\sigma^{*}} \circ \Phi_{\sigma_{*}}(a \times c) .
$$

Recalling the definition (2.7) of $\psi_{s}$, together with the fact that $\mu_{\sigma}$ represents the cross product, one sees at last that the right side of (2.22) reduces to $\partial_{*} \circ \psi_{s}(a \otimes c)$, which confirms (2.11) and completes the proof of Theorem 2.2 .

3. The spectral sequence of a submersion. We now turn to the main task, which is to construct a spectral sequence a spectral sequence associated with a given submersion $f: X \rightarrow Y$. Let $\left\{{ }_{\alpha} p:{ }_{\alpha} E \rightarrow\left|{ }_{\alpha} K\right|\right\}$ be an approximating sequence of simplicial bundles supplied by (1.2), and for each $\alpha$ let $\left\{{ }_{\alpha} E^{r},{ }_{\alpha} d^{r}\right\}$ denote the spectral sequence associated with the corresponding simplicial bundle and given coefficient group by the construction of $\S 2$. Condition (2) in Theorem 1.2 implies that the inclusion ${ }_{\alpha} E \subset{ }_{\beta} E$ (defined for $\alpha<\beta$ ) is filtration preserving, i.e., that ${ }_{\alpha} E_{s} \subset{ }_{\beta} E_{s}$ for all $s$, and consequently induces a homomorphism ${ }_{\alpha}^{\beta} \phi^{r}$ between the respective spectral sequences. This system of 
homomorphisms obviously satisfies the requisite transitivity conditions, and one therefore obtains a direct system of spectral sequences. Taking the direct limits

$$
\begin{aligned}
E^{r} & =\lim _{\alpha} E^{r} \\
d^{r} & =\lim _{\rightarrow} d^{r}
\end{aligned}
$$

gives, for each $r \geqq 1$, a bigraded group $E^{r}$, together with a differential $d^{r}$ of bidegree $(-r, r-1)$. The remaining ingredient of a spectral sequence, i.e., a system of bigraded group isomorphisms $\theta^{r}: H\left(E^{r}\right) \approx E^{r+1}$, can likewise be obtained as a direct limit of the corresponding isomorphisms ${ }_{\alpha} \theta^{r}: H\left({ }_{\alpha} E^{r}\right) \approx{ }_{\alpha} E^{r+1}$. More precisely, the fact that ${ }_{\alpha}^{\beta} \phi^{r}$ constitutes a homomorphism of spectral sequences implies commutativity of the diagram

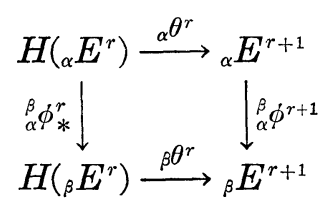

so that $\left\{{ }_{\alpha} \theta^{r}\right\}$ constitutes an isomorphism between the given direct systems of bigraded groups. Since homology commutes with direct limits, one obtains the desired isomorphism $\theta^{r}: H\left(E^{r}\right) \approx E^{r+1}$ by setting

$$
\theta^{r}=\lim _{\alpha} \theta^{r}
$$

We have thus constructed a (first quadrant) $E^{1}$ spectral sequence $\left\{E^{r}, d^{r}\right\}$ associated with the given submersion, and it remains to verify our claims concerning its $E^{2}$ and $E^{\infty}$ terms.

To begin with the $E^{\infty}$ term, we must first exhibit an increasing filtration $F_{s} H_{*}(X ; G)$ of the graded group $H_{*}(X ; G)$, and must subsequently identify $E^{\infty}$ with the bigraded group associated to the given filtration. Consider the filtrations $\left\{F_{s} H_{*}\left({ }_{\alpha} E ; G\right)\right\}$ of $H_{*}\left({ }_{\alpha} E ; G\right)$ defined in 2.1. The inclusions ${ }_{\alpha} E_{s} \subset{ }_{\beta} E_{s}$ induce homomorphisms

$$
{ }_{\alpha}^{\beta} i_{s}: F_{s} H_{*}\left({ }_{\alpha} E ; G\right) \longrightarrow F_{s} H_{*}\left({ }_{\beta} E ; G\right)
$$

which give rise to a commutative diagram

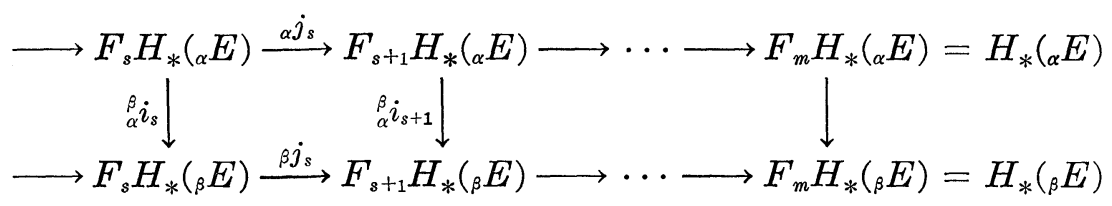

where the $j_{\alpha} j_{s}$ are actual inclusions and $m=\operatorname{dim} Y$. This constitutes a direct system of filtrations, and we define 


$$
\begin{aligned}
F_{s} H_{*}(X ; G) & =\lim _{\rightarrow} F_{s} H_{*}\left({ }_{\alpha} E ; G\right) \\
j_{s} & =\lim _{\alpha} j_{s} .
\end{aligned}
$$

Since the direct limit of monomorphisms is a monomorphism, and since also

$$
\lim _{\rightarrow} H_{*}\left({ }_{\alpha} E ; G\right)=H_{*}(X ; G)
$$

one concludes that $\left\{F_{s} H_{*}(X ; G), j_{s}\right\}$ is a filtration of $H_{*}(X ; G)$. To achieve the desired identification of $E^{\infty}$, we note that for every $\alpha$ there is a short exact sequence

$$
0 \longrightarrow F_{s-1} H_{s+t}\left({ }_{\alpha} E ; G\right) \stackrel{{ }_{\alpha} j_{s-1}}{\longrightarrow} F_{s} H_{s+t}\left({ }_{\alpha} E ; G\right) \stackrel{{ }_{\alpha} \rho}{\longrightarrow}{ }_{\alpha} E_{s, t}^{\infty} \longrightarrow 0 \text {. }
$$

By functoriality of this sequence $[3 ; 3.1$, p. 327] there is a commutative diargram

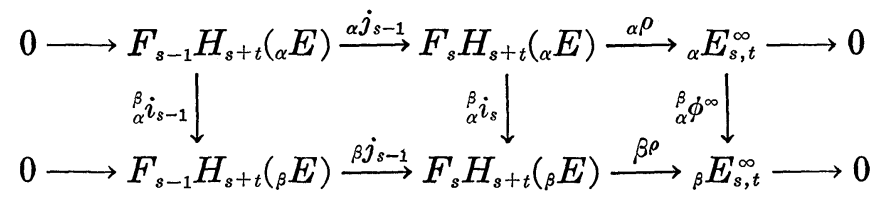

for $\alpha<\beta$, giving a direct system of short exact sequences. Moreover, since $\left\{E^{r}, d^{r}\right\}$ is a convergent spectral sequence, it is obvious that

$$
E^{\infty}=\lim _{\alpha} E^{\infty}
$$

One therefore obtains a short exact sequence

$$
0 \longrightarrow F_{s-1} H_{s+t}(X ; G) \stackrel{j_{s}}{\longrightarrow} F_{s} H_{s+t}(X ; G) \stackrel{\rho}{\longrightarrow} E_{s, t}^{\infty} \longrightarrow 0
$$

in the direct limit, as was to be shown.

It remains to establish an isomorphism

$$
E_{s, t}^{2} \approx H_{s, t}(f ; G)
$$

taking Theorem 2.2 as our starting point. By naturality of the isomorphism [3; 3.1, p. 327]

$$
E_{s, t}^{1} \approx H_{s+t}\left(E_{s}, E_{s-1} ; G\right)
$$

and once again taking into account that homology commutes with direct limits, it will suffice to establish commutativity of the diagram

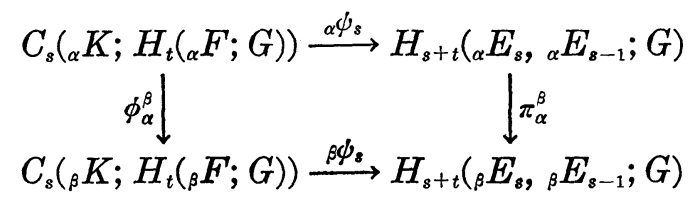


for $\alpha<\beta$, where $\phi_{\alpha}^{\beta}$ is given by (1.11), $\psi_{s}$ by (2.7) and $\pi_{\alpha}^{\beta}$ is inclusion induced. Now this diagram splits into three diagrams, corresponding to the three factors in terms of which $\psi_{s}$ is defined. Taking into account (1.9), (1.10), and (1.11), the first of these subdiagrams becomes

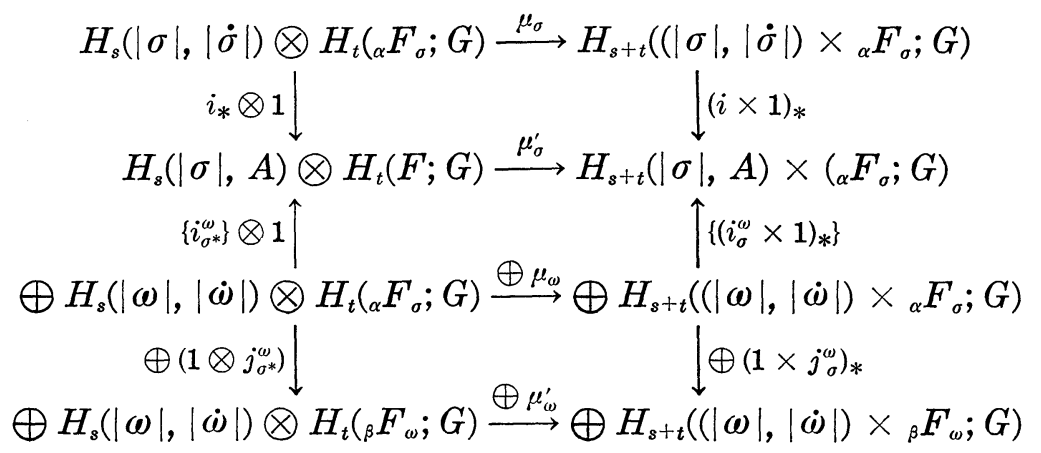

which commutes by naturality of the homology cross product. Next we have the subdiagram

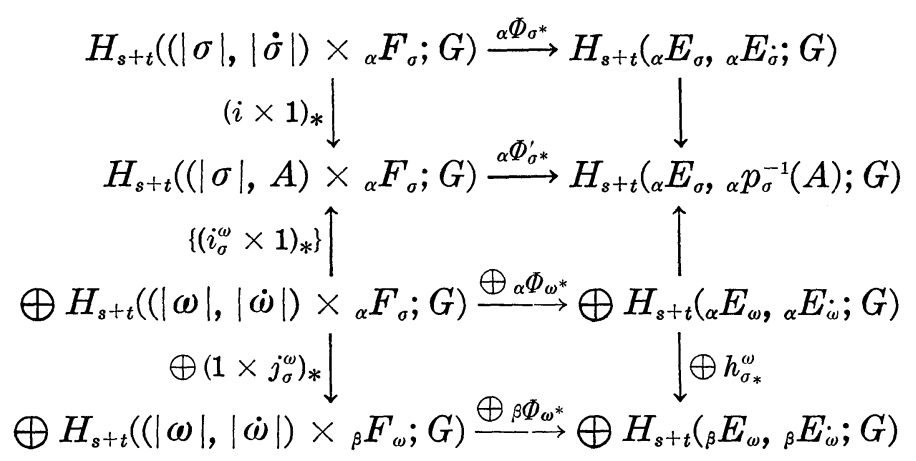

in which the vertical maps on the right are inclusion induced. The top two rectangles derive from commutative diagrams on the space level and consequently commute by functoriality. On the other hand, since the homeomorphisms ${ }_{\alpha} \Phi_{\omega}$ and ${ }_{\beta} \Phi_{\omega}$ derive from independent tubular heighborhood constructions, the space level diagram corresponding to the bottom rectangle need not commute. But once again we are saved by the fact that the space level diagram in question is homotopy commutative; i.e., we shall prove

LeMma 3.1. There exists a homotopy

$$
H:(|\omega|,|\dot{\omega}|) \times{ }_{\alpha} F_{\sigma} \times I \longrightarrow\left({ }_{\beta} E_{\omega},{ }_{\beta} E_{\dot{\omega}}\right)
$$

such that

$$
H: h_{\sigma}^{\omega} \circ{ }_{\alpha} \Phi_{\omega} \cong{ }_{\beta} \Phi_{\omega} \circ j_{\sigma}^{\omega} .
$$

For convenience we will identify the fibers ${ }_{\alpha} F_{\sigma}$ and ${ }_{\beta} F_{\omega}$ with 
${ }_{\alpha} p_{\sigma}^{-1}\left(x_{0}\right)$ and ${ }_{\beta} p_{\omega}^{-1}\left(x_{0}\right)$, respectively, where $x_{0} \in|\omega|$, so that $j_{\sigma}^{\omega}:{ }_{\alpha} F_{\sigma} \rightarrow{ }_{\beta} F_{(\nu}$ becomes an actual inclusion. Moreover, this implies that

$$
{ }_{\alpha} \Phi_{\sigma}\left(x_{0}, y\right)=y
$$

for all $y \in_{\alpha} F_{\sigma}$, and likewise for ${ }_{\beta} \Phi_{\omega}$. Let

$$
\pi:|\omega| \times{ }_{\alpha} F_{\sigma} \longrightarrow{ }_{\alpha} F_{\sigma}
$$

denote the natural projection, and for each $x \in|\omega|$, let $\phi_{x}: I \rightarrow|\omega|$ denote the affine line segment from $x$ to $x_{0}$. The desired homotopy $H$ may now be defined through the formula

$$
H(x, y, t)={ }_{\beta} \Phi_{\omega}\left(x, \pi \circ{ }_{\beta} \Phi_{\omega}^{-1} \circ{ }_{\alpha} \Phi_{\sigma}\left(\phi_{x}(t), y\right)\right) .
$$

It follows immediately that

$$
\begin{aligned}
& H(x, y, 0)={ }_{\alpha} \Phi_{\sigma}(x, y) \\
& H(x, y, 1)={ }_{\beta} \Phi_{\omega}(x, y)
\end{aligned}
$$

and this establishes our lemma.

So far as the third and last subdiagram is concerned (we will not exhibit it), one sees that it is composed entirely of inclusion maps and therefore commutes trivially. This concludes our proof of the following

THEOREM 3.2. Let $f: X \rightarrow Y$ be a submersion and $G$ a coefficient group. There exists a convergent $E^{2}$ spectral sequence with

$$
E_{s, t}^{2} \approx H_{\boldsymbol{s}, t}(f ; G)
$$

and $E^{\infty}$ isomorphic to the bigraded group associated with a filtration of $H_{*}(X ; G)$.

4. Some applications. The applications to be set forth arise by way of our main result 3.2 from the following fact regarding the homology groups $H_{s, t}(f ; G)$.

THEOREM 4.1. Let $f: X \rightarrow Y$ be a submersion and $U$ an open subset of $Y$. If $H_{t}\left(f_{y} ; G\right)=0$ for all $y \notin U$, then

$$
H_{s, t}(f ; G) \approx H_{s, t}(f \mid U ; G),
$$

where $f \mid U$ denotes the restriction of $f$ to the preimage of $U$.

To establish this results, it will be convenient to introduce a few definitions relating to tubular neighborhoods. By a compact tubular neighborhood we shall understand a homeomorphism $\Phi: B \times F \rightarrow V$, 
where $B$ is a compact neighborhood in $Y$ and $V$ a compact subspace of $X$, such that $f \circ \Phi$ is the projection $B \times F \rightarrow B$. Given $y \in B$ we will set $F_{y}=V \cap f_{y}$, and will let

$$
\Phi_{y}^{y^{\prime}}: F_{y} \longrightarrow F_{y},
$$

denote the homeomorphism induced by $\Phi$ for $y, y^{\prime} \in B$.

LemMA 4.2. Let $\Phi: B \times F \rightarrow V$ be a compact tubular neighborhood such that $B$ does not meet $U$, and let $z \subset F_{y}$ be a singular $t$-cycle, where $y \in B$. Then there exists a compact subspace $C \subset X$ such that

$$
\Phi_{y^{\prime}}^{y^{\prime}} z \sim 0 \text { in } C \cap f_{y},
$$

for all $y^{\prime} \in B$.

The proof runs as follows. Our acyclicity assumption implies that for every point $y^{\prime} \in B$ there exists a compact subspace $C_{y^{\prime}}$ such that

$$
\Phi_{y^{\prime}}^{y^{\prime} z} \sim 0 \text { in } C_{y^{\prime}} .
$$

By compactness of $B$ one may conclude with the aid of an elementary tubular neighborhood theorem [7] that there exists a finite sequence of points $y_{1}, \cdots, y_{s}$ and corresponding compact tubular neighborhoods

$$
{ }_{i} \Phi: B_{i} \times F_{i} \longrightarrow V_{i}
$$

such that

(i) each $B_{i}$ is a convex neighborhood of $y_{i}$;

(ii) $\left\{B_{1}, \cdots, B_{s}\right\}$ covers $B$;

(iii) $C_{y_{i}} \subset V_{i}$ for $i=1, \cdots, s$;

(iv) $V \cap f^{-1}\left(B_{i}\right) \subset V_{i}$ for $i=1, \cdots, s$.

We claim now that (4.3) holds with

$$
C=V_{1} \cup \cdots \cup V_{s} .
$$

For let $y^{\prime} \in B$. By (ii) there exists an index $i$ such that $y^{\prime} \in B_{i}$, and by (iii) one has

$$
{ }_{i} \Phi_{y_{i}^{\sharp}}^{y^{\prime}}\left(\Phi_{y^{\sharp}}^{y_{i}} z\right) \sim 0 \quad \text { in } \quad C \cap f_{y^{\prime}} .
$$

Since

$$
\Phi_{y^{\sharp}}^{y^{\prime} z}=\Phi_{y_{i}^{\sharp}}^{y^{\prime}}\left(\Phi_{y^{\sharp}}^{y_{i} z} z\right),
$$

it will suffice to show that

$$
\Phi_{y_{i}}^{y^{\prime}} \cong{ }_{i} \Phi_{y_{i}}^{y^{\prime}} .
$$


But the desired homotopy may be constructed exactly as in Lemma 3.1 , by virtue of conditions (i) and (iv).

We may now proceed to the proof of Theorem 4.1. Let $\left\{U_{\alpha}\right\}$ be a covering of $U$ by an increasing nested sequence of compact sets, and let $\left\{{ }_{\alpha} p:{ }_{\alpha} E \rightarrow\left|{ }_{\alpha} K\right|\right\}$ be an approximating sequence of simplicial bundles satisfying the conditions of Theorem 1.2, together with

$$
\text { for every } \alpha \text { and } \sigma \in_{\alpha} K \text {, if }|\sigma| \text { meets } U_{\alpha} \text {, then }|\sigma| \subset U \text {. }
$$

Let ${ }_{\alpha} \bar{K}$ denote the largest subcomplex of ${ }_{\alpha} K$ such that $\left.\right|_{\alpha} \bar{K} \mid \subset U$, and let ${ }_{\alpha} \bar{p}:\left.{ }_{\alpha} \bar{E} \rightarrow\right|_{\alpha} \bar{K} \mid$ denote the corresponding simplicial bundle. We note that (4.4) implies $\left.U_{\alpha} \subset\right|_{\alpha} K \mid$. One therefore obtains a direct subsystem

$$
\overline{\mathscr{C}}=\left\{C_{*}\left({ }_{\alpha} \bar{K} ; H_{t}\left({ }_{\alpha} \bar{F} ; G\right)\right), \bar{\phi}_{\alpha}^{\beta}\right\}
$$

of chain complexes such that

$$
H_{*}\left(\lim _{\longrightarrow} \overline{\mathscr{C}}\right)=H_{*, t}(f \mid U ; G) .
$$

It will therefore suffice to show that the inclusion

$$
\overline{\mathscr{C}} \subset \mathscr{C} \equiv\left\{C_{*}\left({ }_{\alpha} K ; H_{t}\left({ }_{\alpha} F ; G\right)\right), \phi_{\alpha}^{\beta}\right\}
$$

induces an isomorphism in the direct limit. Since the direct limit of monomorphisms is again a monomorphism, it only remains to check surjectivity. Given a generator

$$
a \otimes c \in H_{s}(|\sigma|,|\dot{\sigma}|) \otimes H_{t}\left({ }_{\alpha} F_{\sigma} ; G\right)
$$

for $\sigma \in_{\alpha} K$, we must show that there exists $\beta>\alpha$ such that

$$
\phi_{\alpha}^{\beta}(a \otimes c) \in C_{s}\left({ }_{\beta} \bar{K} ; H_{t}\left({ }_{\beta} \bar{F} ; G\right)\right) \text {. }
$$

Let $A$ denote the complement of $U$ in $|\sigma|$, and assume without loss of generality that $A$ is nonempty. We may identify ${ }_{\alpha} F_{\sigma}$ with $p_{\sigma}^{-1}(y)$ for some $y \in A$ and let $c$ be represented by a singular $t$-cycle $z \subset p_{o}^{-1}(y)$. Since $A$ is compact, we conclude by Lemma 4.2 that there exists a compact $C \subset X$ such that

$$
{ }_{\sigma} \Phi_{y \sharp z}^{y^{\prime}} \sim 0 \text { in } C \cap f_{y},
$$

for all $y^{\prime} \in A$. Choose $\beta>\alpha$ such that $C \subset{ }_{\beta} E$. Recalling (1.11), one sees that (4.5) may be established by showing that for every $\omega \in_{\beta} K^{\{s\}}$ with $|\omega| \subset|\sigma|$ and $\omega \notin{ }_{\beta} \bar{K}$ one has $j_{\sigma^{*}}^{\omega}(c)=0$. But given such an $\omega$, there must exist a point $y^{\prime} \in|\omega| \cap A$, and one may identify ${ }_{\beta} F_{\omega}$ with $p_{\omega}^{-1}\left(y^{\prime}\right)$. The map $j_{\sigma}^{\omega}:{ }_{\alpha} F_{\sigma} \rightarrow{ }_{\beta} F_{\omega}$ is then represented by ${ }_{\sigma} \Phi_{y}^{y^{\prime}}$ (followed by the inclusion $\left.p_{\sigma}^{-1}\left(y^{\prime}\right) \subset p_{\omega}^{-1}\left(y^{\prime}\right)\right)$, and consequently (4.6) implies the vanishing of $j_{\sigma^{*}}^{\omega}(c)$.

Theorem 4.1 will be of interest in two special cases. In the first 
place, if $f: X^{n} \rightarrow Y^{m}$ is any submersion having connected fibers, the conditions of (4.1) are seen to hold if one takes $U$ to be the subspace of $Y$ corresponding to compact fibers, and takes $t=n-m$. Moreover, the restriction $f \mid U$ will now be a fiber bundle, and if $X, Y$ are both oriented, the homology groups $H_{n-m}\left(f_{y} ; G\right)$ may be canonically identified with $G$ for all $y \in U$. It is therefore easy to evaluate $H_{s, n-m}(f \mid U ; \mathrm{G})$, and one obtains

$$
H_{s, n-m}(f ; G) \approx H_{s}(U ; G) .
$$

Secondly, the conditions of (4.1) may hold for an empty set $U$, in which case one has

$$
H_{s, t}(f ; G)=0 \text { for all } s .
$$

It should also be noted that (4.1) fails when $U$ is not open.

Theorems 3.2 and 4.1 together give rise to an indefinite number of geometric propositions, which we shall illustrate by means of a few examples. The most immediate of these consequences is no doubt the Vietoris-Begle theorem for submersions, which we have previously established [7] by elementary methods. Turning to new results, one has

THeOREM 4.9. Let $f: X \rightarrow Y$ be a submersion between orientable manifolds of dimensions $n$ and $m$, respectively, such that every fiber $f_{y}$ is either a homology $(n-m)$-sphere or else acyclic over a given coefficient group $G$. Then there exists an exact sequence

$$
\cdots \longrightarrow H_{s}(X ; G) \longrightarrow H_{s}(Y ; G) \longrightarrow H_{s-n+m-1}(U ; G) \longrightarrow \cdots
$$

where $U \subset Y$ denotes the subspace corresponding to compact fibers.

This clearly generalizes the Thom-Gysin sequence for sphere bundles, and we also note that for submersions of codimention 1 the result has been previouly established [5] by different methods. To prove our theorem, one concludeds by (3.2) and (4.8) that $E_{s, t}^{2}$ is trivial except when $t$ equals 0 or $(n-m)$, which means that $d^{n-m+2}$ is the only nontrivial differential in our spectral sequence. One therefore obtains exact sequences

$$
0 \longrightarrow E_{s .0}^{\infty} \longrightarrow E_{s_{0}}^{2} \stackrel{d^{n-m+2}}{\longrightarrow} E_{s-n+m-1, n-m}^{2} \longrightarrow E_{s-n+m-1, n-m}^{\infty} \longrightarrow 0
$$

and

$$
0 \longrightarrow E_{s-n+m, n-m}^{\infty} \longrightarrow H_{s}(X ; G) \longrightarrow E_{s, 0}^{\infty} \longrightarrow 0
$$

which by the standard "splicing trick" give rise to a long exact sequence 


$$
\cdots \longrightarrow H_{s}(X ; G) \longrightarrow E_{s, 0}^{2} \longrightarrow E_{s-n+m-1, n-m}^{2} \longrightarrow \cdots
$$

Since

$$
E_{s-n+m-1, n-m}^{2} \approx H_{s-n+m-1}(U ; G)
$$

by (3.2) and (4.7), and clearly

$$
E_{s, 0}^{2} \approx H_{s}(Y ; G)
$$

one obtains the result in question.

It is of interest to note that some of the information contained in the generalized Thom-Gysin sequence for large values of $s$ carries over under much weaker assumptions; for example, one can obtain

Theorem 4.10. Let $f: X \rightarrow Y$ be a submersion between orientable manifolds of dimensions $n$ and $m$, respectively, whose fibers are connected. Then there is an inequality

$$
R_{n-1}(X ; G) \geqq R_{m-1}(U ; G)
$$

involving the Betti numbers of $X$ and $U$, where $U \subset Y$ corresponds to the compact fibers.

To show this we note that $E_{s t}^{2}$ is trivial for $s>m$, and by (4.8) it is also trivial for $t>n-m$. Since all differentials touching $E_{m-1, n-m}^{r}$ are therefore trivial for $r \geqq 2$, one has

$$
E_{m-1, n-m}^{2} \approx E_{m-1, n-m}^{\infty} .
$$

But by (3.2) and (4.7), this implies that

$$
E_{m-1, n-m}^{\infty} \approx H_{m-1}(U ; G),
$$

and the desired rank inequality follows now by the last assertion contained in (3.2).

We will close with another result of this nature, which may be established through a simlar consideration.

THEOREM 4.11. Let $f: X \rightarrow Y$ be as in 4.10, with the additional stipulation that the fibers are G-acyclic in positive dimension $n-m-1$. Then there is an additional rank inequality

$$
R_{n-2}(X ; G) \geqq R_{m-2}(U ; G),
$$

where $U \subset Y$ corresponds to compact fibers.

\section{REFERENCES}

1. S.S. Chern and E. H. Spanier, The homology theory of sphere bundles, Proc. Nat. Acad. Sci., 36 (1950), 248-255. 
2. P. C. Endicott, Simplicial Bundles and the Homology Structure of Submersions, Dissertation, Oregon State University, 1977.

3. S. MacLane, Homology, Springer, 1967.

4. J. Sekino, Homology Theory of Submersions, Dissertation, Oregon State University, 1974.

5. J. W. Smith, An exact sequence for submersions, Bull. Amer. Math. Soc., 74 (1968), 233-236.

6. - Submersions with p-connected fibers, Math. Z., 121 (1971), 288-294.

7. - On the homology structure of submersions, Math. Ann., 193 (1971), 217-224.

8. E. H. Spanier, Algebraic Topology, McGraw-Hill, 1966.

Received August 23, 1978.

Oregon State University

CoRvallis, OR 97331 



\section{PACIFIC JOURNAL OF MATHEMATICS}

\section{EDITORS}

DONALD BABBITT (Managing Editor)

University of California

Los Angeles, CA 90024

HUGo RossI

University of Utah

Salt Lake City, UT 84112

C. C. MOORE and ANDREW OGG

University of California

Berkeley, CA 94720
J. DugundJI

Department of Mathematics

University of Southern California

Los Angeles, CA 90007

R. FinN and J. Milgram

Stanford University

Stanford, CA 94305

ASSOCIATE EDITORS
E. F. BECKENBACH
B. H. NeUmanN
F. WOLF
K. YoSHIDA

\section{SUPPORTING INSTITUTIONS}

UNIVERSITY OF BRITISH COLUMBIA

CALIFORNIA INSTITUTE OF TECHNOLOGY

UNIVERSITY OF CALIFORNIA

MONTANA STATE UNIVERSITY

UNIVERSITY OF NEVADA, RENO

NEW MEXICO STATE UNIVERSITY

OREGON STATE UNIVERSITY

UNIVERSITY OF OREGON
UNIVERSITY OF SOUTHERN CALIFORNIA

STANFORD UNIVERSITY

UNIVERSITY OF HAWAII

UNIVERSITY OF TOKYO

UNIVERSITY OF UTAH

WASHINGTON STATE UNIVERSITY

UNIVERSITY OF WASHINGTON

The Supporting Institutions listed above contribute to the cost of publication of this Journal, but they are not owners or publishers and have no responsibility for its content or policies.

Mathematical papers intended for publication in the Pacific Journal of Mathematics should be in typed form or offset-reproduced, (not dittoed), double spaced with large margins. Please do not use built up fractions in the text of the manuscript. However, you may use them in the displayed equations. Underline Greek letters in red, German in green, and script in blue. The first paragraph or two must be capable of being used separately as a synopsis of the entire paper. Please propose a heading for the odd numbered pages of less than 35 characters. Manuscripts, in triplicate, may be sent to any one of the editors. Please classify according to the scheme of Math. Reviews, Index to Vol. 39. Supply name and address of author to whom proofs should be sent. All other communications should be addressed to the managing editor, or Elaine Barth, University of California, Los Angeles, California, 90024.

50 reprints to each author are provided free for each article, only if page charges have been substantially paid. Additional copies may be obtained at cost in multiples of 50 .

The Pacific Journal of Mathematics is issued monthly as of January 1966. Regular subscription rate: $\$ 84.00$ a year (6 Vols., 12 issues). Special rato: $\$ 42.00$ a year to individual members of supporting institutions.

Subscriptions, orders for numbers issued in the last three calendar years, and changes of address shoud be sent to Pacific Journal of Mathematics, P.O. Box 969, Carmel Valley, CA 93924, U.S.A Old back numbers obtainable from Kraus Periodicals Co., Route 100, Millwood, NY 10546.

PUBLISHED BY PACIFIC JOURNAL OF MATHEMATICS, A NON-PROFIT CORPORATION

Printed at Kokusai Bunken Insatsusha (International Academic Printing Co., Ltd.). 8-8, 3-chome, Takadanobaba, Shinjuku-ku, Tokyo 160, Japan.

Copyright (C) 1980 by Pacific Jounal of Mathematics Manufactured and first issued in Japan 


\section{Pacific Journal of Mathematics \\ Vol. 89, No. $2 \quad$ June, 1980}

Frank Hayne Beatrous, Jr. and R. Michael Range, On holomorphic

approximation in weakly pseudoconvex domains................. 249

Lawrence Victor Berman, Quadratic forms and power series fields ...... 257

John Bligh Conway and Wacław Szymański, Singly generated antisymmetric operator algebras ....................... 269

Patrick C. Endicott and J. Wolfgang Smith, A homology spectral sequence for submersions . . . .................................

Sushil Jajodia, Homotopy classification of lens spaces for one-relator groups with torsion ................................ 301

Herbert Meyer Kamowitz, Compact endomorphisms of Banach

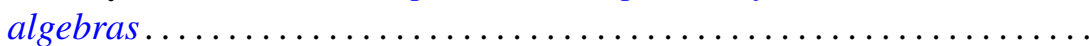

Keith Milo Kendig, Moiré phenomena in algebraic geometry: polynomial

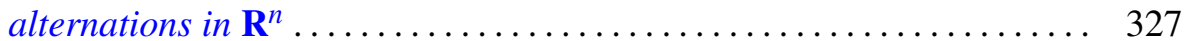

Cecelia Laurie, Invariant subspace lattices and compact operators....... 351

Ronald Leslie Lipsman, Restrictions of principal series to a real form . . . . . 367

Douglas C. McMahon and Louis Jack Nachman, An intrinsic

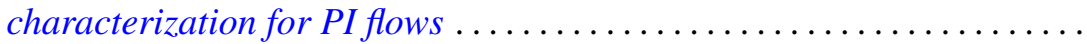

Norman R. Reilly, Modular sublattices of the lattice of varieties of inverse semigroups .................................... 405

Jeffrey Arthur Rosoff, Effective divisor classes and blowings-up of $\mathbf{P}^{2}$ 419

Zalman Rubinstein, Solution of the middle coefficient problem for certain

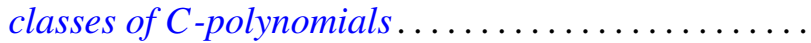

Alladi Sitaram, An analogue of the Wiener-Tauberian theorem for spherical transforms on semisimple Lie groups ................

Hal Leslie Smith, A note on disconjugacy for second order systems ...

J. Wolfgang Smith, Fiber homology and orientability of maps ...

Audrey Anne Terras, Integral formulas and integral tests for series of positive matrices. 